\title{
Breather solutions of the integrable quintic nonlinear Schrödinger equation and their interactions
}

\author{
A. Chowdury, D. J. Kedziora, A. Ankiewicz, and N. Akhmediev \\ Optical Sciences Group, Research School of Physics and Engineering, The Australian National University, Canberra, ACT 2600, Australia
}

(Received 24 November 2014; published 24 February 2015)

\begin{abstract}
We present breather solutions of the quintic integrable equation of the Schrödinger hierarchy. This equation has terms describing fifth-order dispersion and matching nonlinear terms. Using a Darboux transformation, we derive first-order and second-order breather solutions. These include first- and second-order rogue-wave solutions. To some extent, these solutions are analogous with the corresponding nonlinear Schrödinger equation (NLSE) solutions. However, the presence of a free parameter in the equation results in specific solutions that have no analogues in the NLSE case. We analyze new features of these solutions.
\end{abstract}

DOI: 10.1103/PhysRevE.91.022919

PACS number(s): 05.45.Yv, 42.65.Tg, 42.81.Qb

\section{INTRODUCTION}

Soliton interactions form a highly nontrivial part of soliton theory [1-4]. The outcome of interactions between solitons depends upon the particular model used in the theory [5-7]. Due to the great variety of possible interactions, their study can be considered as a separate branch of nonlinear physics. Breathers comprise a different class of solutions of nonlinear evolution equations. Thus, the study of interactions between them can potentially open a new area of research [8,9].

For the nonlinear Schrödinger equation (NLSE), the interaction of breathers was first considered back in 1985 [10]. $N$-breather interactions and their possible practical application were studied later in Ref. [11]. The relation of breathers to rogue wave formation has recently created renewed interest in this subject $[12,13]$. To give an example, the collision of breathers at nonzero angle brings us new unexpected applications [9]. More surprises can be expected when the theory goes beyond the relatively simple NLSE model [14]. In the present work, we extend breather theory to the quintic equation of the nonlinear Schrödinger hierarchy. The sequence of equations in this hierarchy can be found in the classical work of Kano [15]. In reality, it is difficult to study the infinite set of these equations. We can only move step by step in attempting to appreciate the significance of the whole hierarchy.

Such first steps have been made by Hirota [16], who independently found the next lowest-order integrable equation of this hierarchy. This equation contains third-order terms scaled by an independent real parameter. An extension of the hierarchy to fourth-order terms has been made by Lakshmanan, Porsezian, and Daniel [17]. It is presently known as the LPD equation [18,19]. More recently, the quintic equation of the hierarchy has been considered by Hoseini and Marchant [20]. They considered soliton solutions and their interaction. Preliminary studies and a Darboux transformation technique for this equation have been given in our previous work [21]. In the present paper we concentrate on breather solutions of this equation and their interactions. We provide general solutions for the first- and second-order breather solutions and consider several particular cases of interest. For deductive purposes, we separate the cases of purely imaginary and complex eigenvalues.

The fifth-order equation of the nonlinear Schrödinger hierarchy can be written in operator form as:

$$
i \psi_{x}+S[\psi(x, t)]-i \delta Q[\psi(x, t)]=0,
$$

where $S$ is the the second-order nonlinear Schrödinger operator,

$$
S[\psi(x, t)]=\frac{1}{2} \psi_{t t}+\psi|\psi|^{2},
$$

while $Q[\psi(x, t)]$ is the fifth-order quintic operator,

$$
\begin{aligned}
Q[\psi(x, t)]= & \psi_{t t t t t}+10|\psi|^{2} \psi_{t t t}+10\left(\psi\left|\psi_{t}\right|^{2}\right)_{t} \\
& +20 \psi^{*} \psi_{t} \psi_{t t}+30|\psi|^{4} \psi_{t}
\end{aligned}
$$

and $\delta$ is an arbitrary real parameter. Here $x$ is the propagation variable and $t$ is the transverse variable (time in a moving frame). The function $|\psi(x, t)|$ is the envelope of the waves.

Physically, we can think of Eq. (1) as a special case of a more general governing equation for pulse propagation in an optical fiber [22]:

$$
\begin{aligned}
i \psi_{x}= & -\sum_{m=1}^{\infty} \frac{i^{m} \beta_{m}}{m !} \frac{\partial^{m} \psi}{\partial t^{m}}-\gamma\left(1+i s \frac{\partial}{\partial t}\right) \\
& \times\left(\psi \int_{0}^{\infty} R\left(t^{\prime}\right)\left|\psi\left(t-t^{\prime}\right)\right|^{2} d t^{\prime}\right),
\end{aligned}
$$

where the first term on the right-hand side is the expansion of linear dispersion, while the second term describes the nonlinear terms. Here, $s$ is the self-steepening coefficient, the coefficient $\gamma$ is defined by the effective core area and $R(t)$ includes instantaneous and delayed (Raman) contributions of the nonlinear material response. The integral in Eq. (3) is often approximated by taking the series to first-order only, viz. $|\psi|^{2}-\tau_{R} \frac{\partial}{\partial t}\left(|\psi|^{2}\right)$, for Raman delay $\tau_{R}$. However, in reality, we need higher-order terms, and these involve higher order time derivatives of intensity, $|\psi|^{2}$. In experiments, up to quintic terms in this series can be important for pulse durations below $20 \mathrm{ps}$ [23]. Thus, the first term in the operator (2) describes the linear fifth-order dispersion, with $\delta=\beta_{5} / 120$. The other four terms describe the nonlinear dispersion.

\section{BREATHER SOLUTIONS WITH IMAGINARY EIGENVALUES}

The axially aligned Akhmediev breather solutions of the fundamental NLSE have been given explicitly in Refs. [9,13,24-26], while the breather solution for the Hirota equation was firstly presented in Ref. [27]. The basic property of this special solution is that it is localized in ' $x$ ' and periodic in ' $t$ ', making it fundamentally different from soliton solutions. 


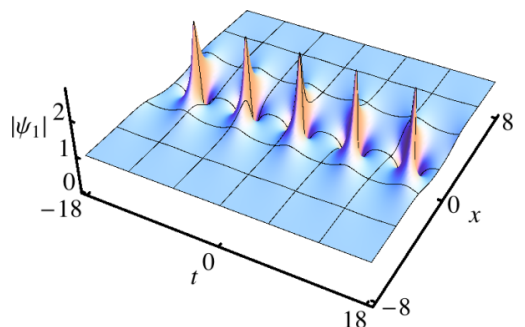

FIG. 1. (Color online) A first-order Akhmediev breather of the quintic equation with eigenvalue $\lambda=0.9 i$. It is given by Eq. (4) with $\delta=\frac{1}{32}$. Here $x$ is the evolution variable and $t$ is the transverse one.

Breathers are closely related to the process of modulation instability of plane waves with periodic perturbations.

Similar breather solutions can be derived for the quintic equation (1). In terms of inverse scattering theory, they correspond to having purely imaginary eigenvalues. They can be obtained in various ways. Our derivation is based upon a Darboux transformation technique given in the Appendix. Following this technique, for the first order, we can write a one-parameter family of breather solutions in the form:

$$
\psi_{1}=\left[\frac{\kappa^{2} \cosh (d x)+2 i d \sinh (d x)}{2\left\{\cosh (d x)-b \cos \left[\kappa\left(t+V_{b} x\right)\right]\right\}}-1\right] e^{i x},
$$

where $\kappa=2 \sqrt{1+\lambda^{2}}$ is the frequency of modulation, $d=$ $\kappa \sqrt{1-\frac{\kappa^{2}}{4}}$ is the growth rate of periodic modulation at the tails of the breather, and $V_{b}=\delta\left(\kappa^{4}-10 \kappa^{2}+30\right)$ defines the skewing angle. Here the eigenvalue is purely imaginary $\lambda=i b$ and $b$ plays the role of the parameter of the family. Thus the frequency of the breather can be written as $\kappa=2 \sqrt{1-b^{2}}$, and consequently the period along the $t$ axis is $T=\pi / \sqrt{1-b^{2}}$. This breather solution is presented in Fig. 1 for the case $b=0.9$. It is identical to the Akhmediev breather $(\mathrm{AB})$ of the NLSE $[8,12,28-30]$, except for the skewed profiles of individual peaks determined by $V_{b}$.

Breathers exist over the range of eigenvalues $0<b<1$. The frequency remains within the interval $0<\kappa<2$. The growth rate, $d$, in (4) is same as in the case of the NLSE [31]. The skewing angle is clearly visible, even for the small value of $\delta=\frac{1}{32}$ that we use here. Parameter $\delta$ is arbitrary and can take large values. The smallness of $\delta$ only signifies the sensitivity of the higher-order terms in the operator $Q[\psi(x, t)]$. Otherwise, variations of $\delta$ do not make qualitative changes in the solution profile apart from skewing and stretching of the localization.

The parameter $V_{b}$ is around 1 when $b \approx 1$ and $\kappa \approx 0$. The effect is relatively large due to the higher-order terms in the quintic operator.

A special case occurs when the eigenvalue $\lambda=i$, i.e., $b \rightarrow$ 1 . The frequency $\kappa$ becomes zero and the period $T$ goes to infinity. The solution (4) becomes undefined as the numerator and denominator are zero. However, taking the limit $b \rightarrow 1$ in the breather solution Eq. (4), we obtain the rogue-wave solution of the quintic equation:

$$
\psi_{1}=\left[\frac{4(1+2 i x)}{4(t+30 \delta x)^{2}+4 x^{2}+1}-1\right] e^{i x} .
$$

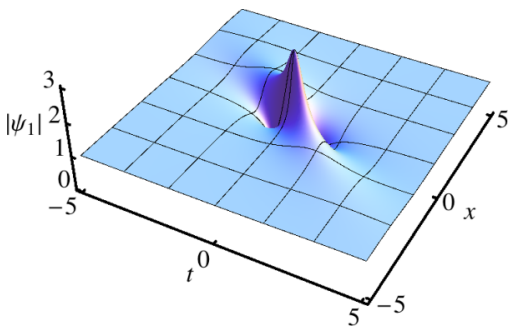

FIG. 2. (Color online) A first-order rogue-wave solution of the quintic equation for $\delta=\frac{1}{32}$.

Only one localized peak remains from the periodic train. This rational solution is presented in Fig. 2. It is an equivalent of the Peregrine solution of the NLSE [32-34]. Indeed, in the limit of $\delta \rightarrow 0$, it is transformed into the actual Peregrine solution.

When the imaginary eigenvalue is above $i$ (i.e., $b>1$ ), in the case of the NLSE, an Akhmediev breather [12,29,33,35,36] is transformed into a Kuznetsov-Ma (KM) soliton [37,38]. A similar transformation occurs in the case of the quintic equation. Indeed, converting the imaginary expressions into real ones in (4), we obtain:

$$
\psi_{m}=\left(\frac{-f^{2} \cos (g x)-2 i g \sin (g x)}{2\left\{\cos (g x)-b \cosh \left[f\left(t+V_{m} x\right)\right]\right\}}-1\right) e^{i x},
$$

where $V_{m}=\delta\left(30+10 f^{2}+f^{4}\right)$, while $g=f b$ and $f=$ $2 \sqrt{b^{2}-1}$. This solution is shown in Fig. 3. It is periodic along the oblique line in the $(x, t)$ plane.

In contrast to the NLSE case, the Kuznetsov-Ma solitons have an oblique angle of propagation due to the $\delta$-dependent velocity component $V_{m}$. This can be clearly seen in Fig. 3 . The soliton propagates along the $x$ axis when $\delta=0$. Each of the three types of breather solutions admits trivial translations along the $x$ and $t$ axes; these are not explicitly shown in the above equations.

\section{TWO-BREATHER SOLUTIONS WITH IMAGINARY EIGENVALUES}

Two-breather solutions depend on two imaginary eigenvalues $\lambda_{1}=i b_{1}$ and $\lambda_{2}=i b_{2}$. They can be obtained in the second step of the Darboux transformation given in Appendix A. The two frequencies $\kappa_{j}$ and eigenvalues are related by $\kappa_{j}=2 \sqrt{1-b_{j}^{2}}$. An example of a solution consisting of two

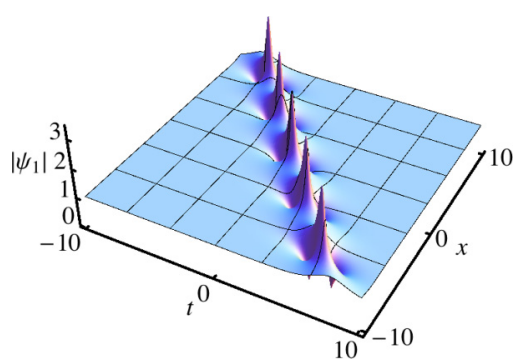

FIG. 3. (Color online) (a) A first-order Kuznetsov-Ma breather of the quintic equation with eigenvalue $\lambda=1.2 i$. It is given by Eq. (6) with $\delta=\frac{1}{64}$. Here $x$ is the evolution variable and $t$ is the transverse one. 


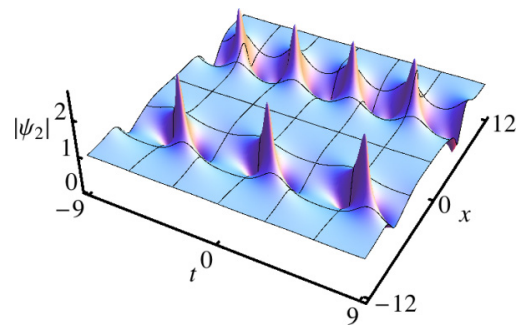

FIG. 4. (Color online) A two-breather solution with eigenvalues $\lambda_{1}=0.65 i$ and $\lambda_{2}=0.85 i$, and $\delta=\frac{1}{32}$. The two breathers are shifted in $x$ due to translations $\Delta x_{1}=5$ and $\Delta x_{2}=-5$.

breathers translated in $x$ by $\Delta x= \pm 5$ is shown in Fig. 4 . The two breathers are well separated and almost independent. They have different periods and skewing angles.

Another example of a two-breather solution is presented in Fig. 5. One imaginary part of the eigenvalues here $\left(b_{1}\right)$ is smaller than 1 while the other one $\left(b_{2}\right)$ is larger than 1. Thus the solution gives a crossing of an $A B$ with a KM soliton. Their periods are defined by the frequencies $\kappa_{1}=2 \sqrt{1-b_{1}^{2}}$ and $f_{2}=2 \sqrt{b_{2}^{2}-1}$. Various combinations are possible. Similar examples of the NLSE breather collisions are given in Ref. [39].

For two ABs located at the same position in $x$, the solution becomes more involved. Patterns depend on the relative frequencies. For example, if the ratio $\kappa_{1}: \kappa_{2}$ is $2: 1$, then the superposition pattern of two $A B s$ is a periodic sequence of merged triplets of Peregrine solutions [40]. If the ratio $\kappa_{1}: \kappa_{2}$ is $3: 2$, the periodic pattern consists of Peregrine pairs alternating with second-order merged triplets [39]. A few examples of superimposed $\mathrm{ABs}$ are shown in Fig. 6. The best way to observe these patterns would be in optical experiments with higher-order modulation instability [41-43].

In Fig. 6(a), the frequency ratio $\kappa_{1}: \kappa_{2}$ is $1: 5$. There are six individual pulse components in each period. Three of them are merged and the other three remain separated. In Fig. 6(b), the ratio of $\kappa_{1}: \kappa_{2}$ is $1: 2$. The pattern consists of a periodic sequence of second-order peaks. When the frequency ratio is close to 1 , the beating period of the pattern increases. This can be clearly seen from Fig. 6(c), where $\kappa_{1}: \kappa_{2}$ is $4: 5$. In the degenerate case, $\kappa_{1}: \kappa_{2}=1$, the beating period is infinite and only one intersecting point between the two breathers remains. A near-degenerate case is shown in Fig. 6(d). Here $\kappa_{1}: \kappa_{2}$ is $9: 10$. Similar patterns appear for second-order Kuznetsov-Ma

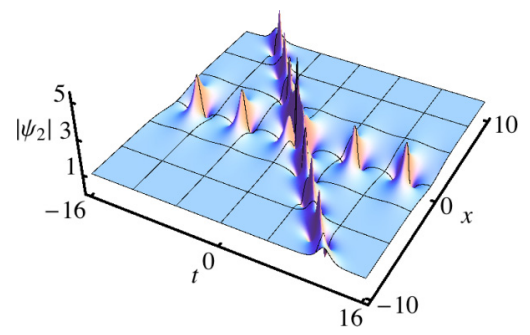

FIG. 5. (Color online) Two-breather solution with one being a Kuznetsov-Ma soliton with eigenvalue $\lambda_{2}=1.35 i$ and the other one being an $\mathrm{AB}$ with $\lambda_{1}=0.85 i$. The parameter $\delta=\frac{1}{64}$.

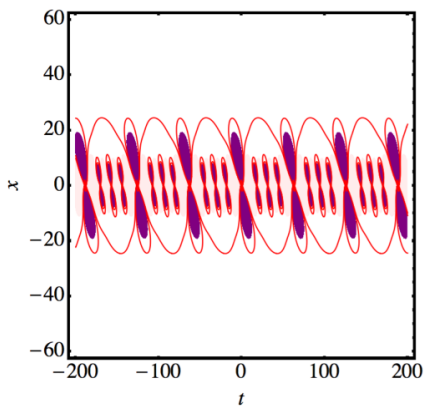

(a)

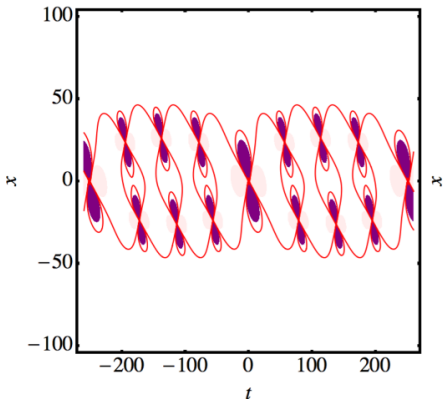

(c)



(b)

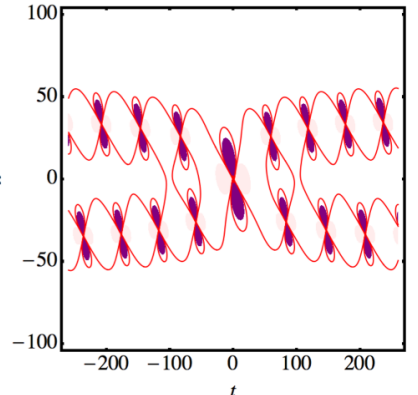

(d)
FIG. 6. (Color online) Contour plots of two superimposed ABs with $\delta=\frac{1}{64}$ and various frequency ratios: (a) $\kappa_{1}=\frac{1}{2}$ and $\kappa_{2}=\frac{1}{10}$; (b) $\kappa_{1}=\frac{1}{5}$ and $\kappa_{2}=\frac{1}{10}$; (c) $\kappa_{1}=\frac{1}{8}$ and $\kappa_{2}=\frac{1}{10} ;$ (d) $\kappa_{1}=\frac{1}{9}$ and $\kappa_{2}=\frac{1}{10}$.

solitons. The frequencies in this case are defined by $i d_{1}$ and $i d_{2}$.

From an experimental point of view, the solutions in Fig. 6 can be excited in the form of higher-order modulation instability, i.e., the same way as in Ref. [42]. Higher frequencies of the instability can be achieved with higher intensities of initial excitation. The specific modeling of terms in the quintic equation can be done by the special design of the optical fiber.

The zero limit of $\kappa_{2}$ transforms one of the ABs into a rogue wave. It can be positioned at any point on the $(x, t)$ plane. An example of a superposition of an $\mathrm{AB}$ with a rogue wave, shifted relative to each other, is shown in Fig. 7. The closed-form solution for this case is given by Eq. (A6) of Appendix A. This diagram is similar to Fig. 3 of Ref. [39] for the NLSE case. For zero shifts, the superposition is shown in Fig. 8. This is an example of a rogue wave on top of an $\mathrm{AB}$.

Taking, additionally, the limit, $\kappa_{1} \rightarrow 0$, we obtain the second-order rogue wave shown in Fig. 9. It has a maximum amplitude of 5 , located at the origin. The solution can be

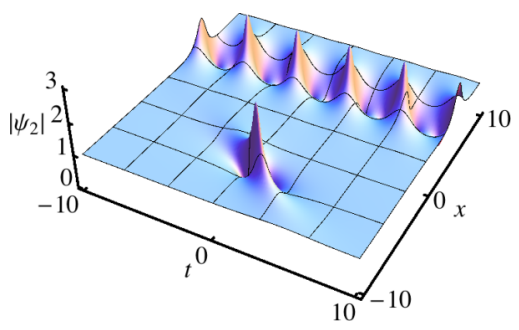

FIG. 7. (Color online) A separated superposition of a rogue wave with an $\mathrm{AB}$ with eigenvalue $\lambda_{1}=0.65 i$ and $\delta=\frac{1}{32}$. 


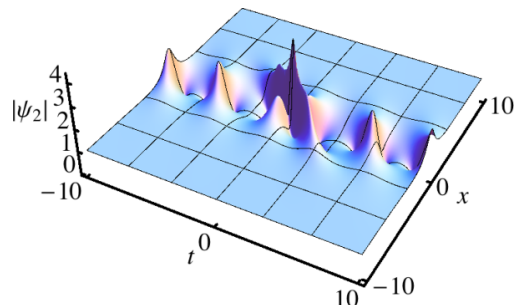

FIG. 8. (Color online) Rogue wave on top of $\mathrm{AB}$ with eigenvalue $\lambda_{1}=0.65 i$ and $\delta=\frac{1}{32}$.

written in the form

$$
\psi=\left[1+\frac{P+i Q}{D}\right] e^{i x},
$$

where

$$
\begin{aligned}
P= & -12\left(-3+72 x^{2}+80 x^{4}+8\left\{2 t^{4}+3 t^{2}\left(1+4 x^{2} \Gamma\right)\right.\right. \\
& +240 t^{3} x \delta+900 x^{2} \delta^{2}\left[19+12 x^{2}\left(1+150 \delta^{2}\right)\right] \\
& \left.\left.+60 t x \delta\left[11+12 x^{2}\left(1+300 \delta^{2}\right)\right]\right\}\right), \\
Q= & -24 x\left\{-15+8 x^{2}+8\left[-3 t^{2}+2 t^{4}+4 t^{2} x^{2}+2 x^{4} \Gamma^{2}\right.\right. \\
& \left.\left.+60 t x\left(5+4 t^{2}+4 x^{2} \Gamma\right) \delta+900\left(13+12 t^{2}\right) x^{2} \delta^{2}\right]\right\}, \\
D= & +64 t^{6}+64 x^{6} \Gamma^{3}+11520 t^{5} x \delta \\
& -432 x^{4}\left(-1+300 \delta^{2}\right)\left(1+2900 \delta^{2}\right) \\
& +36 x^{2}\left(11+37900 \delta^{2}\right)+720 t x \delta[25 \\
& \left.+8 x^{2}\left(5+2 x^{2} \Gamma^{2}-6300 \delta^{2}\right)\right]+1920 t^{3} x \delta[-5 \\
& \left.+12 x^{2}\left(1+1500 \delta^{2}\right)\right]+48 t^{4}\left[1+4 x^{2}\left(1+4500 \delta^{2}\right)\right] \\
& +12 t^{2}\left\{9+8 x^{2}\left[-3+2 x^{2} \Gamma+900\left(-13+10 x^{2} \Gamma\right) \delta^{2}\right]\right\},
\end{aligned}
$$

and where $\Gamma=1+900 \delta^{2}$. The solution has a variable equation parameter $\delta$ that controls the skew angle of the pattern. If $\delta=0$, this solution coincides with the second-order Akhmediev-Peregrine solution of the NLSE, presented earlier in Refs. [10,44]. It has been experimentally observed in hydrodynamics [45].

\section{DEGENERATE TWO-BREATHER SOLUTION}

The common inverse scattering technique [46] does not allow two eigenvalues to be equal. When this happens, the solution is undefined. It needs additional effort to unveil such solutions in explicit form [47]. In order to do that, we rewrite the solution, making the change $\kappa_{2} \rightarrow \kappa_{1}+h$. Then, we take

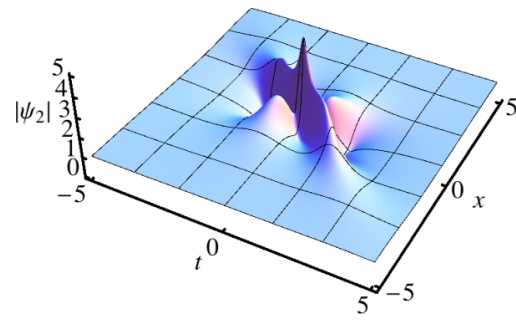

FIG. 9. (Color online) A second-order quintic rogue wave with $\delta=\frac{1}{64}$ given by Eq. (7).



(a)

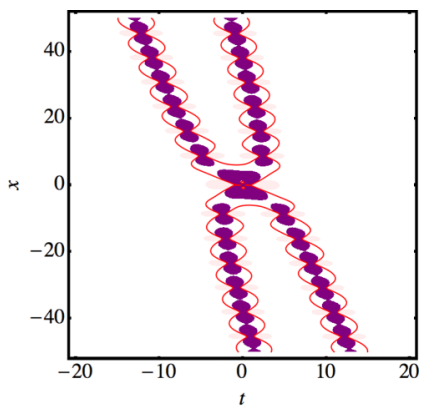

(b)
FIG. 10. (Color online) Degenerate two-breather solutions. (a) A two-AB solution with $\kappa=0.8$ and $\delta=\frac{1}{64}$. (b) A two-KM soliton solution with $\kappa=0.8 i$ and $\delta=\frac{1}{256}$.

a Taylor series expansion of this expression in terms of $h$ and retain only the lowest-order terms. The resulting solution becomes well defined. Solutions obtained in this way are known as degenerate solutions [47].

In our case, the resulting solution contains only one frequency $\kappa_{1}$, which we denote as $\kappa$. This solution is given by Eq. (A7) in the Appendix. It is a combination of rational, hyperbolic and trigonometric functions of $x$ and $t$. The free parameter of the solution is $\kappa$. Two illustrations are given in Fig. 10. One case is for real $\kappa=0.8$, while the other one is for imaginary $\kappa=0.8 i$. The former case is a superposition of two $\mathrm{ABs}$ while the latter case is a superposition of two KM solitons with equal eigenvalues. In each case, there is only one point of crossing between the two breathers. For the NLSE case, when $\delta=0$, the solutions have been given earlier in Refs. $[39,47,48]$. In the NLSE case, the solutions have mirror symmetry relative to the axes $x$ and $t$. For the quintic equation, this symmetry is lost. Instead, there is an inversion symmetry relative to the origin.

\section{ROGUE-WAVE TRIPLET}

A second-order rogue-wave solution can have additional free parameters, which split the superposition into its fundamental components. For the NLSE case, such a solution has been found in Ref. [49] and studied in detail in Ref. [40]. Surprisingly, the second-order rogue-wave solution is split into three fundamental rogue waves. Each of them is a Peregrine solution. This splitting feature is different from higher-order soliton solutions. The second-order soliton solution consists of two solitons.

A similar splitting can be observed in the case of the secondorder rogue-wave solution of the quintic equation. In order to derive this solution, we have to take into account translations that may depend on $\kappa$. Namely, we represent the translations in the form of a series in $\kappa$ :

$$
x_{j}=\sum_{m=1}^{N} \kappa^{2(m-1)} X_{m j}, \quad t_{j}=\sum_{m=1}^{N} \kappa^{2(m-1)} T_{m j},
$$

with $1 \leqslant j \leqslant m$. At the very point $\kappa=0$, the translations are given by $X_{11}, T_{11}, X_{12}$, and $T_{12}$. If these are zero, the solution is centered at the origin. However, the coefficients of 
higher-order terms in these series become the free parameters of the solution when we take the exact limit of $\kappa \rightarrow 0$. The number, $N$, of terms needed in these series, depends upon the order of the solution. For the second-order solution, two lowest-order terms are sufficient. For the solution centered at the origin, this leaves us with four parameters: $X_{21}, T_{21}, X_{22}$, and $T_{22}$. These coefficients enter the solution as the differences $x_{d}=X_{22}-X_{21}$ and $t_{d}=T_{22}-T_{21}$. Thus, only these two free independent parameters are involved in the solution:

$$
\psi=\left[1+\frac{G_{t r}+i H_{t r}}{D_{t r}}\right] e^{i x},
$$

where

$$
\begin{aligned}
G_{t r}= & -12\left\{-3+16 t^{4}+24 t^{2}\left(1+4 x^{2} \Gamma\right)+1920 t^{3} x \delta+8 x^{2}\left[9+10 x^{2} \Gamma+900\left(19+2 x^{2} \Gamma\right) \delta^{2}\right]\right. \\
& \left.+480 t x \delta\left[11+12 x^{2}\left(1+300 \delta^{2}\right)\right]-128(t+30 x \delta) t_{d}-128\left(x+30 t \delta+900 x \delta^{2}\right) x_{d}\right\}, \\
H_{t r}= & -24\left\{x \left[-15+16 t^{4}+1920 t^{3} x \delta+480 t x\left(5+4 x^{2} \Gamma\right) \delta+8 t^{2}\left[-3+4 x^{2}\left(1+2700 \delta^{2}\right)\right]\right.\right. \\
& \left.\left.+8 x^{2}\left(1+11700 \delta^{2}+2 x^{2} \Gamma^{2}\right)\right]-128 x(t+30 x \delta) t_{d}+16\left(1+4 t^{2}-4 x^{2} \Gamma\right) x_{d}\right\}, \\
D_{t r}= & +64 t^{6}+64 x^{6} \Gamma^{3}+11520 t^{5} x \delta-432 x^{4}\left(-1+300 \delta^{2}\right)\left(1+2900 \delta^{2}\right)+36 x^{2}\left(11+37900 \delta^{2}\right) \\
& +1920 t^{3} x \delta\left[-5+12 x^{2}\left(1+1500 \delta^{2}\right)\right]+d 48 t^{4}\left[1+4 x^{2}\left(1+4500 \delta^{2}\right)\right]+12 t^{2} \\
& \times\left\{9+8 x^{2}\left[-3+2 x^{2} \Gamma+900\left(-13+10 x^{2} \Gamma\right) \delta^{2}\right]\right\}+720 t x \delta\left[25+8 x^{2}\left(5-6300 \delta^{2}+2 x^{2} \Gamma^{2}\right)\right] \\
& +128\left\{8 t_{d}^{2}+x_{d}\left[x\left(-9+12 t^{2}-4 x^{2}\right)+30 t\left(-3+4 t^{2}+12 x^{2} \Gamma\right) \delta+900\left(-19+12 t^{2}\right) x \delta^{2}+3240000 x^{3} \delta^{4}\right.\right. \\
& \left.\left.+8 \Gamma x_{d}\right]+t_{d}\left[4 t^{3}+360 t^{2} x \delta+30 x \delta\left[-19+12 x^{2}\left(-1+300 \delta^{2}\right)\right]+3 t\left(-1+4 x^{2}\left[-1+900 \delta^{2}\right]\right)+480 \delta x_{d}\right]\right\},
\end{aligned}
$$

and $\Gamma=1+900 \delta^{2}$

An example of this solution is shown in Fig. 11. It is a triplet of first-order rogue waves similar to the rogue wave triplet of the NLSE [40]. For zero values of $x_{d}$ and $t_{d}$, the three components merge and the solution reduces to Eq. (7). In the NLSE case, the individual components of the triplet are located at the vertices of an equilateral triangle [40]. The quintic terms shift these relative positions. The triangle becomes skewed in the $(x, t)$ plane, in addition to the skewing of each component of the solution.

\section{TWO-BREATHER SOLUTIONS WITH COMPLEX EIGENVALUES}

The one-soliton solution of the NLSE with complex eigenvalue $\lambda=a+i b$ has an amplitude $b$ and velocity $a$. Thus, the inclusion of a real part of $\lambda$ results in the soliton propagating at an angle to the $x$ axis. A one-breather solution with a complex eigenvalue has the same property. It has a propagation direction that is oblique to the $x$ axis [9,13,24-26]. Velocity appears in the one-breather solutions of the quintic equation, as well. However, there is an additional tilt to this direction caused by the quintic terms. This can be seen from

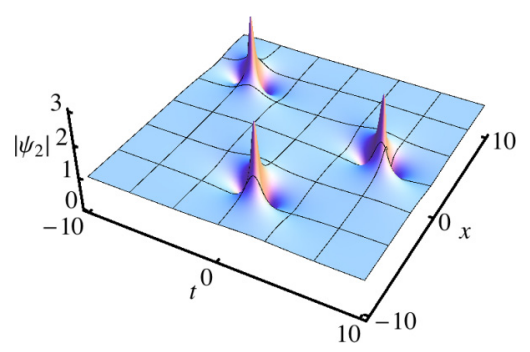

FIG. 11. (Color online) Quintic rogue wave triplet given by Eq. (8) with $x_{d}=-25, t_{d}=-50$ and $\delta=\frac{1}{64}$.
Fig. 3. Even the Kuznetsov-Ma soliton with $a=0$, which would be propagating along the $x$ axis in the case of the NLSE, has a nonzero tilt for the quintic equation.

The explicit form of the one-breather solution is:

$$
\psi_{1}=\left[1+2 b \frac{G_{1}+i H_{1}}{D_{1}}\right] e^{i x},
$$

where the functions $G_{1}, H_{1}$, and $D_{1}$ are combinations of trigonometric and hyperbolic functions:

$$
\begin{aligned}
G_{1}= & \cos \left(x V_{T}+t \kappa_{r}\right) \cosh \left(2 \chi_{i}\right) \\
& -\cosh \left(x V_{H}+t \kappa_{i}\right) \sin \left(2 \chi_{r}\right), \\
H_{1}= & \cos \left(2 \chi_{r}\right) \sinh \left(x V_{H}+t \kappa_{i}\right) \\
& +\sin \left(x V_{T}+t \kappa_{r}\right) \sinh \left(2 \chi_{i}\right), \\
D_{1}= & \cosh \left(x V_{H}+t \kappa_{i}\right) \cosh \left(2 \chi_{i}\right) \\
& -\cos \left(x V_{T}+t \kappa_{r}\right) \sin \left(2 \chi_{r}\right),
\end{aligned}
$$

with

$$
\begin{aligned}
& \kappa=2 \sqrt{1+\lambda^{2}}=\kappa_{r}+i \kappa_{i} \\
& \chi=\frac{1}{2} \cos ^{-1}\left(\frac{\kappa}{2}\right)=\chi_{r}+i \chi_{i}
\end{aligned}
$$

and

$$
\begin{aligned}
V_{T} & =-b \kappa_{i}+a \kappa_{r}+\delta\left(\Omega \kappa_{i}+\mho \kappa_{r}\right) \\
V_{H} & =a \kappa_{i}+b \kappa_{r}+\delta\left(\mho \kappa_{i}-\Omega \kappa_{r}\right) \\
\Omega & =16 a b\left(1-4 a^{2}+4 b^{2}\right) \\
\mho & =2\left[3+8 a^{4}+4 b^{2}+8 b^{4}-4 a^{2}\left(1+12 b^{2}\right)\right] .
\end{aligned}
$$

Again, if we take $\delta=0$, Eq. (9) reduces to the breather solution of the NLSE with complex eigenvalue, presented in Ref. [13]. The one-breather solution is similar to the breather solution given in Fig. 3. However, the direction of propagation of the 


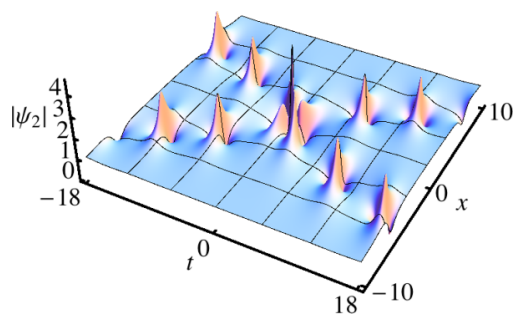

FIG. 12. (Color online) Two-breather collision. The two complex eigenvalues are $\lambda_{1}=0.05+0.9 i$ and $\lambda_{2}=-0.05+0.9 i$. The parameter $\delta=\frac{1}{64}$.

whole breather and the skewing of each peak are now defined by the expressions $V_{H} / \kappa_{i}$ and $V_{T} / \kappa_{r}$, respectively.

Having the one-breather solution, and using Darboux transformations, we can construct two-breather solutions, as described in the Appendix. One example is shown in Fig. 12. In analogy with multisoliton solutions, this pattern can be called a collision of two breathers. The choice of parameters is such that the maximum of collision is located at the origin. However, translation of the components may convert the maximum into zero. Except for the area of the collision, the two breathers are roughly the same as given by Eq. (9). In addition, there are small shifts imposed at the sides of each breather by the collision.

A collision of general type is shown in Fig. 12. An interesting possibility is the alignment of the two breathers along the same direction in the $(x, t)$ plane. Having an additional parameter, $\delta$, in the quintic equation, such an alignment can be arranged without equalizing the real parts of the two eigenvalues. Below, we consider various special cases of this solution that are possible only for the quintic equation. These cases do not have analogues among the solutions of the NLSE equation.

Indeed, we now consider the case when the directions of propagation of the two colliding breathers coincide. This happens if

$$
\frac{V_{H 1}}{\kappa_{1 i}}=\frac{V_{H 2}}{\kappa_{2 i}} .
$$

The notation here is the same as in Eq. (9), with the addition of a subscript that corresponds to a particular breather, one or two. For example, $\lambda_{j}=a_{j}+i b_{j}$, where $j=1,2$. Similarly:

$$
\begin{aligned}
V_{T j} & =-b_{j} \kappa_{j i}+a_{j} \kappa_{j r}+\delta\left(\Omega_{j} \kappa_{j i}+\mho_{j} \kappa_{j r}\right) \\
V_{H j} & =a_{j} \kappa_{j i}+b_{j} \kappa_{j r}+\delta\left(\mho_{j} \kappa_{j i}-\Omega_{j} \kappa_{j r}\right) \\
\Omega_{j} & =16 a_{j} b_{j}\left(1-4 a_{j}^{2}+4 b_{j}^{2}\right), \\
\mho_{j} & =2\left[3+8 a_{j}^{4}+4 b_{j}^{2}+8 b_{j}^{4}-4 a_{j}^{2}\left(1+12 b_{j}^{2}\right)\right] .
\end{aligned}
$$

With this notation, the condition (10) can be written as:

$$
\begin{aligned}
& {\left[a_{1} \kappa_{1 i}+b_{1} \kappa_{1 r}+\delta\left(\mho_{1} \kappa_{1 i}-\Omega_{1} \kappa_{1 r}\right)\right] / \kappa_{1 i}} \\
& \quad=\left[a_{2} \kappa_{2 i}+b_{2} \kappa_{2 r}+\delta\left(\mho_{2} \kappa_{2 i}-\Omega_{2} \kappa_{2 r}\right)\right] / \kappa_{2 i} .
\end{aligned}
$$

This algebraic equation cannot be solved analytically. However, we can solve it numerically. Figure 13 shows the locus of points on the complex plane of $\lambda_{1}$ for fixed $\lambda_{2}$ and $\delta$, found by solving Eq. (11). The plot demonstrates a rich variety of possibilities that lead to the parallel superimposed



FIG. 13. (Color online) Locus of points on the plane of complex eigenvalues $\lambda_{1}$ that lead to parallel breathers. The second eigenvalue is fixed and equal to $\lambda_{2}=0.08+0.9 i$ for solid (blue) lines and equal to $\lambda_{2}=0.08+1.5 i$ for the dashed (red) lines. Parameter $\delta=\frac{1}{64}$. Solid red dots, blue rectangles, green triangles, and magenta stars on the curves are chosen to signify the examples in the figures below.

breathers. Indeed, the locus of points is a set of continuous curves of solutions rather than isolated points. In addition, there are three such curves. Consequently, we can have a rich variety of specific two-breather solutions. By choosing different points on the curves, we can have various patterns.

To begin, we solve the two-breather alignment condition for the specific values of eigenvalues $\lambda_{1}=a_{1}+0.8 i$ with $a_{1}$ to be identified and $\lambda_{2}=0.08+0.9 i$. The plot in Fig. 13 provides three real solutions for $a_{1}$ shown by the solid red dots. They are: $a_{1}=-1.70693, a_{1}=0.141238$ and $a_{1}=1.1181$. These three values of $a_{1}$ correspond to three qualitatively different cases. They are shown in three panels of Fig. 14.



(a)

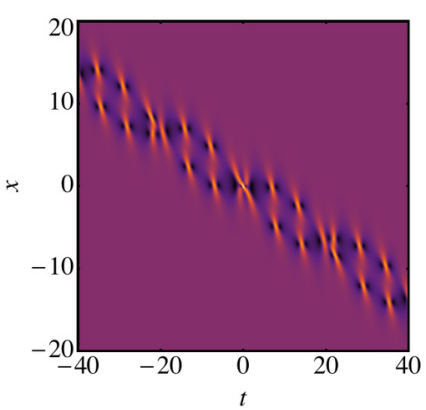

(b)

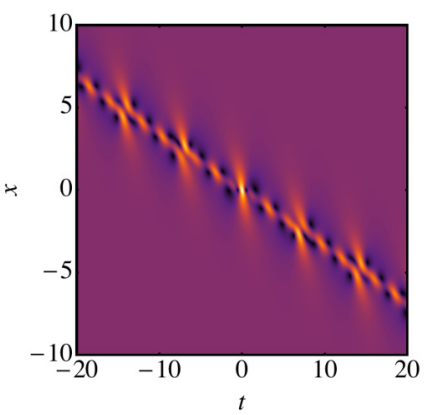

(c)

FIG. 14. (Color online) Two-breather superposition with the condition Eq. (11), marked by solid red dots in Fig. 13. Here $\lambda_{2}=0.08+0.9 i$ and $\delta=\frac{1}{64}$. (a) $\lambda_{1}=-1.70693+0.8 i$; (b) $\lambda_{1}=$ $0.141238+0.8 i ;$ (c) $\lambda_{1}=1.1181+0.8 i$. 
Figure 14(a) shows the complex pattern of two superimposed breathers with different periods. In this case, we observe the beating on top of the original periods of the two breathers. The transverse widths of the two breathers are comparable. This results in a single-mode pattern along the combined breather. The peaks of the breather remain single peaks. They are higher at the points of constructive interference of the two components with radiation around them.

Figure 14(b) shows the beating pattern of two breathers for $\lambda_{1}=0.141238+0.8 i$. The periods of two breathers are incommensurate. Consequently, the beating period is not well pronounced. Significant differences in the widths of the two first-order breathers set up a complex multimode transverse pattern of the superposition. The skew angles $V_{T} / \kappa_{r}$ of the two breathers are also different, and this is an additional reason for the complex pattern.

In Figure 14(c), the beating period is comparable with the periods of each breather. The widths of the two components are also slightly different. Thus, we have another complex pattern different from the two previous cases. The highest peak in each of the three superpositions is always at the point $(0,0)$. This is due to the fact that individual breathers are centered at the origin. If we add translations, the two breathers can be separated in space and time.

We can use another value of $\lambda_{2}$ when solving Eq. (11). For example, the dashed curves in Fig. 13 are calculated for $\lambda_{2}=0.08+1.5 i$. If we choose $b_{1}=0.9$, we again obtain three solutions for $a_{1}$. These are: $a_{1}=-1.71834, a_{1}=0.200181$, and $a_{1}=0.990488$. Two of these points are shown by the green triangles on the dashed red curves.

Figure 15 shows the patterns of breather superposition for two of the above solutions, $a_{1}=-1.71834$ and $a_{1}=$ 0.990488 . There are no fundamental differences between these composite breathers and those shown in Fig. 14. The patterns remain quasiperiodic along the breather. The angles of propagation in the $(x, t)$ plane vary, of course. Clearly, we can observe a wide variety of patterns, taking into account that the condition of Eq. (11) admits an infinite number of possibilities.

Among all of them, we can choose some special cases. Namely, solving Eq. (11), we have the chance to select equal values $b_{1}$ and $b_{2}$. For example, if the eigenvalue $\lambda_{2}=$

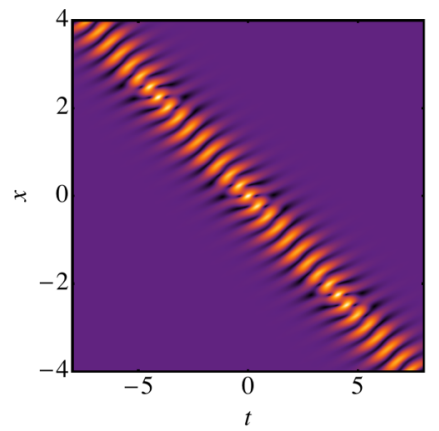

(a)

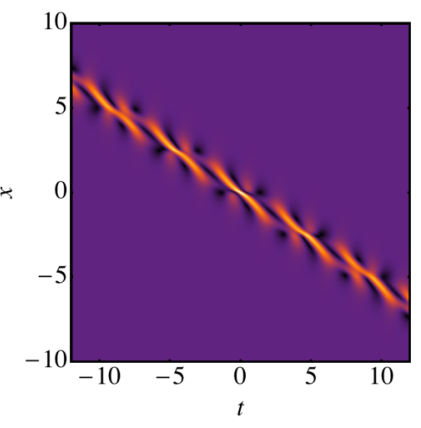

(b)
FIG. 15. (Color online) Two-breather superposition with the condition of Eq. (11), marked by green triangles in Fig. 13. Here, $\lambda_{2}=0.08+1.5 i$ and $\delta=\frac{1}{64}$. (a) $\lambda_{1}=-1.71834+0.9 i$; (b) $\lambda_{1}=$ $0.990488+0.9 i$

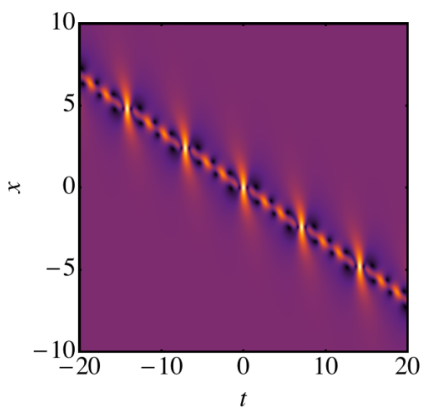

(a)

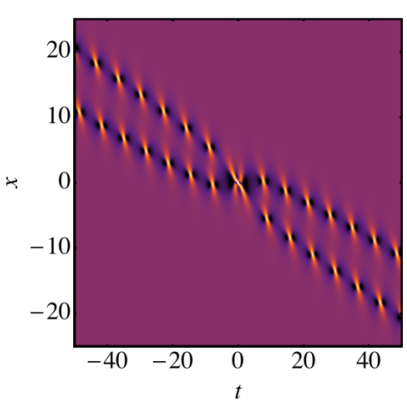

(b)
FIG. 16. (Color online) Two-breather superposition with the condition of Eq. (11), marked by blue rectangles in Fig. 13. Here (a) $\lambda_{1}=1.22549+0.9 i, \lambda_{2}=0.08+0.9 i$; (b) $\lambda_{1}=0.08+0.9 i$, $\lambda_{2}=0.08+0.9 i$ (degenerate case). Parameter $\delta=\frac{1}{64}$.

$0.08+0.9 i$ is chosen, three eigenvalues $\lambda_{1}$ satisfy Eq. (11). They are: $\lambda_{1}=-1.77613+0.9 i, \lambda_{1}=0.08+0.9 i$, and $\lambda_{1}=$ $1.22549+0.9 i$. Two of them are shown by blue rectangles in Fig. 13. Clearly, the second of these eigenvalues coincides with $\lambda_{2}$ and the two-breather solution is then degenerate. Two other choices show similar patterns, as before, except for the completely periodic pattern along the breather. This happens due to the equal imaginary parts of the two eigenvalues $b_{1}=b_{2}$. One example is shown in Fig. 16(a). The other choice, $\lambda_{1}=-1.77613+0.9 i$, provides a similar periodic beating pattern. However, the eigenvalue $\lambda_{1}=0.08+0.9 i$ results in the degenerate solution shown in Fig. 16(b). The two breathers split, apart from the origin, which is the point of their collision. This pattern is similar to degenerate solutions considered in the previous sections.

Special cases can be found for other values of $\lambda_{2}$. For example, if we choose $\lambda_{2}=0.08+1.5 i$, we obtain four values for the first eigenvalue: $\lambda_{1}=-2.29479+1.5 i, \lambda_{1}=0.08+1.5 i$, $\lambda_{1}=0.273178+1.5 i$, and $\lambda_{1}=1.93937+1.5 i$ that satisfy Eq. (11). The second of these solutions, $\lambda_{1}=0.08+1.5 i$, corresponds to the degenerate case. The pattern of this solution (not shown here) is similar to the case shown in Fig. 10(b). However, two other patterns for $\lambda_{1}=-2.29479+1.5 i$ and $\lambda_{1}=0.273178+1.5 i$ are shown in Figs. 17(a) and 17(b), respectively. They correspond to the magenta stars in Fig. 13.

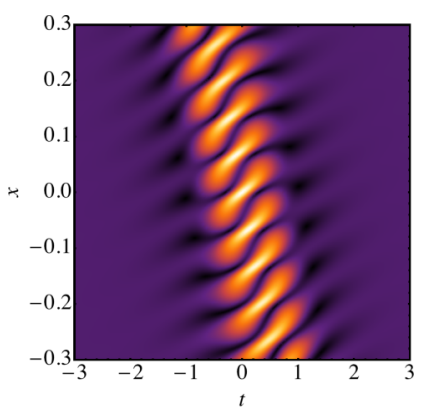

(a)



(b)
FIG. 17. (Color online) Two-breather superposition with the condition Eq. (11), marked by magenta stars in Fig. 13. Here, $\lambda_{2}=0.08+1.5 i$ and $\delta=\frac{1}{64}$. (a) $\lambda_{1}=-2.29479+1.5 i$; (b) $\lambda_{1}=$ $0.273178+1.5 i$. 
These figures demonstrate once again the huge variety of possibilities in designs of two-breather solutions. Each of these patterns is strictly periodic along the breather, because of the equal imaginary parts of the eigenvalues.

\section{CONCLUSION}

Here, we have presented breather solutions of the quintic integrable equation of the Schrödinger hierarchy. This equation has terms describing fifth-order dispersion and additional matching nonlinear terms with a free real parameter $\delta$. These terms transform the breathers of the NLSE, adding a skewing angle into the breather patterns.

Significant differences from the NLSE case can be observed for second-order breathers. In particular, two breathers can be aligned in parallel without matching their eigenvalues. These beating superpositions generate an infinite variety of patterns. Some of them have been illustrated graphically, and conditions for their appearance have been discussed in detail.

Practical realization of new solutions in optical fibers will require special arrangements. First, we need to operate with pulses with durations below $20 \mathrm{ps}$ in order to activate quintic terms in the fiber [23]. This, in turn, will require higher intensities of the optical radiation. The use of specially designed fibers is another requirement that may help to obtain a special relation between the higher-order terms. On the bright side, we have the exact solutions that can be used for preparing initial conditions in the experiments.

The remaining question to answer is the robustness of the new solutions. Several papers $[9,50,51]$ already posed and partially answered this question for the breather solutions of the NLSE. The answer for the solutions of higher-order equations is still needs to be given. We leave this for future work.

\section{ACKNOWLEDGMENTS}

The authors acknowledge the support of the Australian Research Council (Discovery Project No. DP140100265). N.A. and A.A. acknowledge support from the Volkswagen Stiftung. A.C. acknowledges Endeavour Postgraduate Award support.

\section{APPENDIX: DETAILS OF DARBOUX TRANSFORMATION TECHNIQUE}

\section{One- and two-breather solutions with imaginary eigenvalues and limiting cases}

The Darboux transformation technique has been developed in general form by Matveev and Salle [52], and applied for breather solutions of the NLSE in Ref. [11]. The quintic integrable equation requires a few modifications in comparison to the NLSE case. These are related to the inclusion of quintic terms into the $2 \times 2$ matrices of the Lax-pair formalism. Multibreather solutions require the use of a plane wave as the seeding solution of the technique [11]. This is different from the multisoliton solutions, which only require the zero solution in order to start the process [52].

Substituting the seeding plane wave $\psi_{0}=e^{i x}$ and the eigenvalue $\lambda_{1,2}$ into the set of linear differential equations relative to $r$ and $s$ (compatibility condition) and solving them, at the first step, we obtain the following functions

$$
\begin{gathered}
r_{1, j}=-2 i e^{-i x / 2} \cos \left(A_{j}+i B_{j}+\chi_{j}\right) \\
s_{1, j}=2 e^{i x / 2} \sin \left(A_{j}+i B_{j}-\chi_{j}\right)
\end{gathered}
$$

where, depending on the eigenvalue that we use, $j=1$ or 2 . The variables in this solution are

$$
\begin{aligned}
A_{j} & =d_{j} G_{j} x_{s j}+\kappa_{j} t_{s j} / 2+\frac{\pi}{4}, \quad B_{j}=d_{j} x_{s j} / 2, \\
\chi_{j} & =\frac{1}{2} \cos ^{-1}\left(\frac{\kappa_{j}}{2}\right), \quad G_{j}=V_{j} / v_{j} \\
V_{j} & =\delta\left(\kappa_{j}^{4}-10 \kappa_{j}^{2}+30\right), \quad v_{j}=\sqrt{4-\kappa_{j}^{2}} \\
d_{j} & =\frac{1}{2} \kappa_{j} v_{j}, \quad \kappa_{j}=2 \sqrt{1+\lambda_{j}^{2}} .
\end{aligned}
$$

When we admit translations along $x$ and $t$, we use the shifted spatial $x_{s j}=x-x_{j}$ and temporal $t_{s j}=t-t_{j}$ variables. In this section, we use the purely imaginary eigenvalue, $\lambda_{j}=i b_{j}$.

Solutions of the quintic equation, at the first and second steps of calculation, can be found from the same equation

$$
\psi_{n}=\psi_{n-1}+\frac{2\left(\lambda_{n}^{*}-\lambda_{n}\right) s_{n, 1} r_{n, 1}^{*}}{\left|r_{n, 1}\right|^{2}+\left|s_{n, 1}\right|^{2}},
$$

where $n$ is the order of the solution. In particular, it provides the one- and the two-breather solutions of the quintic equation.

The main advantage of the Darboux transformation technique is that the second-order linear functions $r_{2,1}$ and $s_{2,1}$ do not require solution of differential equations. Instead, we use recursive algebraic relations derived in Ref. [52]. A simple diagram for such calculations is given in Fig. 2.2 of Ref. [47] (see also Ref. [11]). The calculations require a knowledge of $r_{1,1}, s_{1,1}, r_{1,2}$ and $s_{1,2}$ from Eqs. (A1) and (A2). Thus, we obtain

$$
\begin{aligned}
r_{2,1}= & \frac{1}{D_{2}}\left\{-2 \cos \left(A_{2}+i B_{2}+\chi_{2}\right)\left[b_{1} \cos \left(2 A_{1}\right)\right.\right. \\
& \left.\times \cos \left(2 \chi_{1}\right)-b_{2} \cosh \left(2 B_{1}\right)+b_{2} \sin \left(2 A_{1}\right) \sin \left(2 \chi_{1}\right)\right] \\
& -4 b_{1} \cos \left(A_{1}+i B_{1}+\chi_{1}\right) \sin \left(A_{1}-i B_{1}-\chi_{1}\right) \\
& \left.\times \sin \left(A_{2}+i B_{2}-\chi_{2}\right)\right\}
\end{aligned}
$$

$$
\begin{aligned}
s_{2,1}= & \frac{1}{D_{2}}\left\{-2 i b_{1} \cos \left(A_{2}+i B_{2}+\chi_{2}\right) \sin \left(2 A_{1}\right)\right. \\
& +2 i\left[b_{1} \cos \left(2 A_{1}\right) \cos \left(2 \chi_{1}\right)+b_{2} \cosh \left(2 B_{1}\right)\right. \\
& \left.-b_{2} \sin \left(2 A_{1}\right) \sin \left(2 \chi_{1}\right)\right] \sin \left(A_{2}+i B_{2}-\chi_{2}\right) \\
& \left.+2 b_{1} \cos \left(A_{2}+i B_{2}+\chi_{2}\right) \sinh \left(2\left(B_{1}+i \chi_{1}\right)\right)\right\},
\end{aligned}
$$

where $D_{2}=\cosh \left(2 \mathrm{~B}_{1}\right)-\sin \left(2 \mathrm{~A}_{1}\right) \sin \left(2 \chi_{1}\right)$. Substituting these $r_{2,1}$ and $s_{2,1}$ into Eq. (A3), we obtain the two-breather solution of quintic equation. The calculations are trivial and not presented here.

The case when $\lambda_{2} \rightarrow i$ is special. In this case $\kappa_{2} \rightarrow 0$, the period of breather becomes infinite and the breather is transformed into a rogue wave. After applying L'Hôpital's 
rule to the ratio,the second-order solution takes the form:

$$
\psi=\left[1+\frac{N_{k}}{D_{k}}\right] e^{i x},
$$

where

$$
\begin{aligned}
N_{k}= & \kappa_{1}^{2}\left\{-8 t_{s 2}\left(t_{s 2}+60 \delta x_{s 2}\right)\left[\cosh \left(2 B_{1}\right)-\sin \left(2 A_{1}\right) \sin \left(\chi_{1}\right)\right]-v_{1}\left[\sin \left(2 A_{1}\right)\right.\right. \\
& \left.\left.+i \sinh \left(2 B_{1}+i \chi_{1}\right)\right]\left(1+4 y^{2}+4 x_{s 2}^{2}\right)-2\left[\cosh \left(2 B_{1}\right)-\sin \left(2 A_{1}\right) \sin \left(\chi_{1}\right)\right]\left[-1+4 x_{s 2}\left(-i+x_{s 2}+900 \delta^{2} x_{s 2}\right)\right]\right\},
\end{aligned}
$$

and

$$
\begin{aligned}
D_{k}= & 4 v_{1}\left\{4 \cos \left(\chi_{1}\right)\left[y \cos \left(2 A_{1}\right)-x_{s 2} \sinh \left(2 B_{1}\right)\right]+\left[\cosh \left(2 B_{1}\right) \sin \left(\chi_{1}\right)-\sin \left(2 A_{1}\right)\right]\left(1-4 y^{2}-4 x_{s 2}^{2}\right)\right\} \\
& -\left[\cosh \left(2 B_{1}\right)-\sin \left(2 A_{1}\right) \sin \left(\chi_{1}\right)\right]\left(1+4 y^{2}+4 x_{s 2}^{2}\right)\left(-8+\kappa_{1}^{2}\right) .
\end{aligned}
$$

As expected, it is a combination of rational, trigonometric, and hyperbolic functions.

\section{Degenerate two-breather solution: $\kappa_{1}=\kappa_{2}=\kappa$}

When the two eigenvalues $\lambda_{1}$ and $\lambda_{2}$ coincide, the solution again becomes undefined. It has to be recovered by using L'Hôpital's rule on the numerator and denominator of the resulting expression. In this way, we obtain:

$$
\psi=\left[1+\frac{N_{d}}{D_{d}}\right] e^{i x},
$$

where $N_{d}$ and $D_{d}$ are

$$
\begin{aligned}
N_{d}= & -4 \kappa c_{2}\{-2[\kappa(-(-3 \cosh (2 B)+\cosh (2(B+i \chi))) \sin (2 A)+(-2+\cos (4 A)) \sin (\chi)+i \sinh (4 B+i \chi)) \\
& \left.+2 \cos (2 A) \cos (\chi) \cosh (2 B+i \chi) y_{1}\left(t+5 x \delta y_{2}\right)\right]+2 c_{2}[\cos (4 A) \cos (\chi)+\cosh (4 B+i \chi) \\
& \left.\left.-2 i \sin (2 A) \sinh (2 B)+2 x \cos (\chi)(-i+\sin (2 A) \sinh (2 B+i \chi)) y_{3}\right]\right\}, \\
D_{d}= & 2\left\{\left(8-3 \kappa^{2}\right) \cos (4 A)+8 \cosh (4 B)+\kappa^{2}\left[3-2 \cos (2 \chi) \sin ^{2}(2 A)\right]+8 \cosh (2 B)\right. \\
& \times\left[c_{1} t \cos (2 A) \cos (\chi)-\kappa^{2} \sin (2 A) \sin (\chi)\right]-8 x\left(8-6 \kappa^{2}+\kappa^{4}\right) \cos (\chi) \sin (2 A) \sinh (2 B) \\
& \left.+4 \cos (\chi)\left[10 c_{1} x \delta \cos (2 A) \cosh (2 B) y_{2}+\cos (\chi) y_{1}\left(y_{1}\left(t+5 x \delta y_{2}\right)^{2}-x^{2} y_{3}^{2}\right)\right]\right\} .
\end{aligned}
$$

Here $c_{1}=v^{3}, \quad v=c_{2}=\sqrt{4-\kappa^{2}}, d=\frac{1}{2} \kappa v$,

$$
\begin{aligned}
A & =\frac{1}{4}[\pi+2(t+V x) \kappa], \quad B=\frac{d x}{2}, \\
V & =\delta\left(30-10 \kappa^{2}+\kappa^{4}\right), \quad \chi=\cos ^{-1}\left(\frac{\kappa}{2}\right), \\
y_{1} & =\kappa^{2}-4, \quad y_{2}=6-6 \kappa^{2}+\kappa^{4}, y_{3}=\kappa^{2}-2 .
\end{aligned}
$$

\section{One- and two-breather solutions with complex eigenvalues}

The two-breather solution of the NLSE has been presented earlier in Ref. [13]. Here, we follow our previous calculations, given explicitly in Appendixes A and B of Ref. [13] and modify them for the case of quintic equation. As the eigenvalues $\lambda_{j}=a_{j}+i b_{j}$ are complex numbers, the parameters $\kappa_{j}=$ $2 \sqrt{1+\lambda_{j}^{2}}$ and $\chi_{j}=\cos ^{-1}\left(\frac{\kappa_{j}}{2}\right)$ are now also complex: $\kappa=$ $\kappa_{j r}+i \kappa_{j i}$ and $\chi=\chi_{j r}+i \chi_{j i}$. The linear functions $r$ and $s$ of the first step are

$$
r_{1,1}=2 i e^{-i x / 2} \sin (G), \quad s_{1,1}=2 e^{i x / 2} \cos (H) .
$$

The arguments of the trigonometric functions can also be written explicitly as complex functions: $G=A_{r}+i A_{i}$ and $H=B_{r}+i B_{i}$, where the real and imaginary parts are

$$
\begin{aligned}
A_{r} & =\chi_{1 r}+\frac{1}{2}\left(\kappa_{1 r} t+d_{1 r} x\right)-\frac{\pi}{4} \\
A_{i} & =\chi_{1 i}+\frac{1}{2}\left(\kappa_{1 i} t+d_{1 i} x\right) \\
B_{r} & =-\chi_{1 r}+\frac{1}{2}\left(\kappa_{1 r} t+d_{1 r} x\right)-\frac{\pi}{4} \\
B_{i} & =-\chi_{1 i}+\frac{1}{2}\left(\kappa_{1 i} t+d_{1 i} x\right) .
\end{aligned}
$$

Here $d_{1}=d_{1 r}+i d_{1 i}$ is the complex coefficient of the argument $x$. Its components $d_{1 r}$ and $d_{1 i}$ can be written in the form:

$$
\begin{aligned}
& d_{1 r}=-b_{1} \kappa_{1 i}+a_{1} \kappa_{1 r}+\delta\left(\Omega_{1} \kappa_{1 i}+\mho_{1} \kappa_{1 r}\right), \\
& d_{1 i}=a_{1} \kappa_{1 i}+b_{1} \kappa_{1 r}+\delta\left(\mho_{1} \kappa_{1 i}-\Omega_{1} \kappa_{1 r}\right),
\end{aligned}
$$

where

$$
\begin{aligned}
& \Omega_{1}=16 a_{1} b_{1}\left(1-4 a_{1}^{2}+4 b_{1}^{2}\right), \\
& \mho_{1}=2\left[3+8 a_{1}^{4}+4 b_{1}^{2}+8 b_{1}^{4}-4 a_{1}^{2}\left(1+12 b_{1}^{2}\right)\right] .
\end{aligned}
$$


Substituting the functions $r_{1,1}$ and $s_{1,1}$ above into Eq. (A3), we obtain the one-breather solution with complex eigenvalue $\lambda_{1}$ :

$$
\psi=\left[1+\frac{8}{D_{1}} i b_{1} \cosh \left(B_{i}-i B_{r}\right) \sinh \left(A_{i}+i A_{r}\right)\right] e^{i x},
$$

where $D_{1}=\cos \left(2 B_{r}\right)+\cosh \left(2 A_{i}\right)+\cosh \left(2 B_{i}\right)-\cos \left(2 A_{r}\right)$. This solution can be transformed into the expression (9) of the main text.

For the two-breather solution, the second-order linear functions $r_{1,2}$ and $s_{1,2}$ are

$$
r_{1,2}=2 i e^{-i x / 2} \sin (C), \quad s_{1,2}=2 e^{i x / 2} \cos (D),
$$

where $C=C_{r}+i C_{i}$ and $D=D_{r}+i D_{i}$ are complex arguments with the components:

$$
\begin{aligned}
& C_{r}=\chi_{2 r}+\frac{1}{2}\left(\kappa_{2 r} t+d_{2 r} x\right)-\frac{\pi}{4} \\
& C_{i}=\chi_{2 i}+\frac{1}{2}\left(\kappa_{2 i} t+d_{2 i} x\right) \\
& D_{r}=-\chi_{2 r}+\frac{1}{2}\left(\kappa_{2 r} t+d_{2 r} x\right)-\frac{\pi}{4} \\
& D_{i}=-\chi_{2 i}+\frac{1}{2}\left(\kappa_{2 i} t+d_{2 i} x\right),
\end{aligned}
$$

and $d_{2}=d_{2 r}+i d_{2 i}$ is the complex coefficient of the argument $x$ with the components:

$$
\begin{aligned}
& d_{2 r}=-b_{2} \kappa_{2 i}+a_{2} \kappa_{2 r}+\delta\left(\Omega_{2} \kappa_{2 i}+\mho_{2} \kappa_{2 r}\right) \\
& d_{2 i}=a_{2} \kappa_{2 i}+b_{2} \kappa_{2 r}+\delta\left(\mho_{2} \kappa_{2 i}-\Omega_{2} \kappa_{2 r}\right) .
\end{aligned}
$$

Here

$$
\begin{aligned}
& \Omega_{2}=16 a_{2} b_{2}\left(1-4 a_{2}^{2}+4 b_{2}^{2}\right) \\
& \mho_{2}=2\left[3+8 a_{2}^{4}+4 b_{2}^{2}+8 b_{2}^{4}-4 a_{2}^{2}\left(1+12 b_{2}^{2}\right)\right] .
\end{aligned}
$$

The second-order linear functions $r_{2,1}^{*}$ and $s_{2,1}$ are calculated using $r_{1,1}, s_{1,1}, r_{1,2}$, and $s_{1,2}$, as shown in the diagram Fig. 2.2 in Ref. [47]. They are:

$$
\begin{aligned}
r_{2,1}^{*}= & -\frac{2}{D_{1}} e^{i x / 2}\left(4 i b_{1} \cosh \left(B_{i}-i B_{r}\right) \cosh \left(D_{i}+i D_{r}\right)\right. \\
& \times \sinh \left(A_{i}+i A_{r}\right)+\sinh \left(C_{i}+i C_{r}\right) \\
& \times\left\{\cosh \left(2 A_{i}\right)\left[-a_{1}+a_{2}+i\left(b_{1}-b_{2}\right)\right]\right. \\
& +\cos \left(2 A_{r}\right)\left[a_{1}-a_{2}-i\left(b_{1}-b_{2}\right)\right] \\
& \left.\left.+\left[\cos \left(2 B_{r}\right)+\cosh \left(2 B_{i}\right)\right]\left[-a_{1}+a_{2}-i\left(b_{1}+b_{2}\right)\right]\right\}\right) \\
s_{2,1}= & -\frac{2}{D_{1}} e^{i x / 2}\left(4 i b_{1} \cosh \left(B_{i}-i B_{r}\right) \sinh \left(A_{i}+i A_{r}\right)\right. \\
& \times \sinh \left(C_{i}-i C_{r}\right)+\cosh \left(D_{i}-i D_{r}\right) \\
& \times\left\{\cosh \left(2 A_{i}\right)\left[a_{1}-a_{2}-i\left(b_{1}+b_{2}\right)\right]\right. \\
& +\cos \left(2 A_{r}\right)\left[-a_{1}+a_{2}+i\left(b_{1}+b_{2}\right)\right] \\
& \left.\left.+\left[\cos \left(2 B_{r}\right)+\cosh \left(2 B_{i}\right)\right]\left[a_{1}-a_{2}+i\left(b_{1}-b_{2}\right)\right]\right\}\right) .
\end{aligned}
$$

Substituting these expressions into Eq. (A3), we obtain the two-breather solution. It is omitted here, as the calculations are trivial. When $\delta=0$, this solution coincides with the solution of Eq. (22) given in Appendix B of Ref. [13].
[1] D. Turaev, A. G. Vladimirov, and S. Zelik, Long-Range Interaction and Synchronization of Oscillating Dissipative Solitons, Phys. Rev. Lett. 108, 263906 (2012).

[2] R. Ganapathy, K. Porsezian, A. Hasegawa, and V. N. Serkin, Soliton interaction under soliton dispersion management, IEEE J. Quantum Electronics 44, 383 (2008).

[3] M. G. Clerc, S. Coulibaly, N. Mujica, R. Navarro, and T. Sauma, Soliton pair interaction law in parametrically driven newtonian fluid, Phil. Trans. R. Soc. A 367, 3213 (2009).

[4] P. Peterson, T. Soomere, J. Engelbrecht, and E. van Groesen, Soliton interaction as a possible model for extreme waves in shallow water, Nonlinear Proc. Geophys. 10, 503 (2003).

[5] E. Ibragimov, A. A. Struthers, D. J. Kaup, J. D. Khaydarov, and K. D. Singer, Three-wave interaction solitons in optical parametric amplification, Phys. Rev. E 59, 6122 (1999).

[6] G. Biondini, K.-I. Maruno, M. Oikawa, and H. Tsuji, Soliton interactions of the Kadomtsev-Petviashvili equation and generation of large-amplitude water waves, Stud. Appl. Math. 122, 377 (2009)

[7] M. J. Ablowitz and D. E. Baldwin, Nonlinear shallow oceanwave soliton interactions on flat beaches, Phys. Rev. E 86, 036305 (2012).

[8] B. Frisquet, B. Kibler, and G. Millot, Collision of Akhmediev breathers in nonlinear fiber optics, Phys. Rev. X 3, 041032 (2013).
[9] V. E. Zakharov and A. A. Gelash, Nonlinear stage of modulation instability, Phys. Rev. Lett. 111, 054101 (2013).

[10] N. Akhmediev, V. M. Eleonskii, and N. E. Kulagin, Generation of periodic trains of picosecond pulses in an optical fiber: Exact solutions, Sov. Phys. JETP 62, 894 (1985).

[11] N. Akhmediev, V. I. Korneev, and N. V. Mitskevich, $\mathrm{N}$-modulation signals in a single-mode optical waveguide under nonlinear conditions, Sov. Phys. JETP 67, 89 (1988).

[12] K. B. Dysthe and K. Trulsen, Note on breather type solutions of the NLS as models for freak-waves, Physica Scripta T82, 48 (1999).

[13] N. Akhmediev, J. M. Soto-Crespo, and A. Ankiewicz, Extreme waves that appear from nowhere: on the nature of rogue waves, Phys. Lett. A 373, 2137 (2009).

[14] U. Bandelow and N. Akhmediev, Persistence of rogue waves in extended nonlinear Schrödinger equations: Integrable SasaSatsuma case, Phys. Lett. A 376, 1558 (2012).

[15] T. Kano, Normal form of nonlinear Schrödinger equation, J. Phys. Soc. Japan 58, 4322 (1989).

[16] R. Hirota, Exact envelope-soliton solutions of a nonlinear wave equation, J. Math. Phys. 14, 805 (1973).

[17] M. Lakshmanan, K. Porsezian, and M. Daniel, Effect of discreteness on the continuum limit of the Heisenberg spin chain, Phys. Lett. A 133, 483 (1988). 
[18] K. Porsezian, M. Daniel, and M. Lakshmanan, On the integrability aspects of the one-dimensional classical continuum isotropic biquadratic Heisenberg spin chain, J. Math. Phys. 33, 1807 (1992).

[19] K. Porsezian, Completely integrable nonlinear Schrödinger type equations on moving space curves, Phys. Rev. E 55, 3785 (1997).

[20] S. M. Hoseini and T. R. Marchant, Solitary wave interaction and evolution for a higher-order hirota equation, Wave Motion 44, 92 (2006)

[21] A. Chowdury, D. J. Kedziora, A. Ankiewicz, and N. Akhmediev, Soliton solutions of an integrable nonlinear Schrödinger equation with quintic terms, Phys. Rev. E 90, 032922 (2014).

[22] Govind P. Agrawal, Nonlinear fiber optics: Its history and recent progress [invited], JOSA B 28, A1 (2011).

[23] S. Backus, C. G. Durfee III, G. Mourou, H. C. Kapteyn, and M. M. Murnane, 0.2-tw laser system at 1khz, Opt. Lett. 22, 1256 (1997).

[24] M. Tajiri and Y. Watanabe, Breather solutions to the focusing nonlinear Schrödinger equation, Phys. Rev. E 57, 3510 (1998).

[25] Q-Han Park and H. J. Shin, Parametric control of soliton light traffic by cw traffic light, Phys. Rev. Lett. 82, 4432 (1999).

[26] Lu Li, Zhonghao Li, Shuqing Li, and Guosheng Zhou, Modulation instability and solitons on a cw background in inhomogeneous optical fiber media, Opt. Commun. 234, 169 (2004).

[27] N. Akhmediev, V. I. Korneev, and N. V. Mitskevich, Modulation instability of a continuous signal in an optical fiber taking into account third-order dispersion, Izvestiya Vysshikh Uchebnykh Zavedenii, Radiofizika 33, 111 (1990).

[28] Ch. Mahnke and F. Mitschke, Possibility of an Akhmediev breather decaying into solitons, Phys. Rev. A 85, 033808 (2012).

[29] N. V. Priya, M. Senthilvelan, and M. Lakshmanan, Akhmediev breathers, Ma solitons, and general breathers from rogue waves: A case study in the Manakov system, Phys. Rev. E 88, 022918 (2013).

[30] A. Bendahmane, A. Mussot, P. Szriftgiser, O. Zerkak, G. Genty, J. M. Dudley, and A. Kudlinski, Experimental dynamics of Akhmediev breathers in a dispersion varying optical fiber, Opt. Lett. 39, 4490 (2014).

[31] N. Akhmediev and V. I. Korneev, Modulation instability and periodic solutions of nonlinear schrödinger equation, Teor. Mat. Fiz. 69, 189 (1986).

[32] D. H. Peregrine, Water waves, nonlinear Schrödinger equations and their solutions, J. of the Australian Math. Soc. Series B. Appl. Math. 25, 16 (1983).

[33] V. I. Shrira and V. V. Geogjaev, What makes the Peregrine soliton so special as a prototype of freak waves?, J. Eng. Math. 67, 11 (2010).

[34] B. Kibler, J. Fatome, Ch. Finot, G. Millot, F. Dias, G. Genty, N. Akhmediev, and J. M. Dudley, The peregrine soliton in nonlinear fiber optics, Nature Phys. 6, 790 (2010).
[35] K. Hammani, B. Wetzel, B. Kibler, J. Fatome, Ch. Finot, G. Millot, N. Akhmediev, and J. M. Dudley, Spectral dynamics of modulation instability described using Akhmediev breather theory, Opt. Lett. 36, 2140 (2011).

[36] M. Erkintalo, G. Genty, B. Wetzel, and J. M. Dudley, Akhmediev breather evolution in optical fiber for realistic initial conditions, Phys. Lett. A 375, 2029 (2011).

[37] Y.-C. Ma, The perturbed plane-wave solutions of the cubic Schrödinger equation, Studies in Applied Math. 60, 43 (1979).

[38] B. Kibler, J. Fatome, C. Finot, G. Millot, G. Genty, B. Wetzel, N. Akhmediev, F. Dias, and J. M. Dudley, Observation of Kuznetsov-Ma soliton dynamics in optical fiber, Sci. Rep. 2, 463 (2012).

[39] D. J. Kedziora, A. Ankiewicz, and N. Akhmediev, Secondorder nonlinear Schrödinger equation breather solutions in the degenerate and rogue wave limits, Phys. Rev. E 85, 066601 (2012).

[40] A. Ankiewicz, D. J. Kedziora, and N. Akhmediev, Rogue wave triplets, Phys. Lett. A 375, 2782 (2011).

[41] S. Wabnitz and N. Akhmediev, Efficient modulation frequency doubling by induced modulation instability, Opt. Commun. 283, 1152 (2011).

[42] M. Erkintalo, K. Hammani, B. Kibler, Ch. Finot, N. Akhmediev, J. M. Dudley, and G. Genty, Higher-order modulation instability in nonlinear fibre optics, Phys. Rev. Lett. 107, 253901 (2011).

[43] Feng-De Zong, Yu-Sheng Yan, and Sen-Ting Shen, Higher-order modes of modulation instability in Bose-Einstein condensates with a time-dependent three-dimensional parabolic potential, J. Phys. Soc. Japan 83, 104002 (2014).

[44] N. Akhmediev, A. Ankiewicz, and M. Taki, Waves that appear from nowhere and disappear without a trace, Phys. Lett. A 373, 675 (2009).

[45] A. Chabchoub, N. Hoffmann, M. Onorato, and N. Akhmediev, Super rogue waves: Observation of a higher-order breather in water waves, Phys. Rev. X 2, 011015 (2012).

[46] V. E. Zakharov and A. B. Shabat, Exact theory of twodimensional self-focusing and one-dimensional self-modulation of waves in nonlinear media, J. Exp. Theor. Phys. 34, 62 (1972).

[47] N. Akhmediev and A. Ankiewicz, Solitons: nonlinear pulses and beams (Chapman and Hall, London, 1997).

[48] N. Akhmediev and A. Ankiewicz, Spatial Soliton X-Junctions and Couplers, Opt. Commun. 100, 186 (1993).

[49] P. Dubard, P. Gaillard, C. Klein, and V. B. Matveev, On NLS multi-rogue waves solutions and positon solutions of the $\mathrm{KdV}$ equation, Eur. Phys. J. Special Topic 185, 247 (2010).

[50] Adrian Ankiewicz, N. Devine, and Nail Akhmediev, Are rogue waves robust against perturbations?, Phys. Lett. A 373, 3997 (2009).

[51] Robert A. Van Gorder, Orbital Instability of the Peregrine Soliton, J. Phys. Soc. Jpn. 83, 054005 (2014).

[52] V. B. Matveev and M. A. Salle, Darboux transformations and solitons (Springer, Berlin, 1991). 